\title{
DISTANCIAMENTO SOCIAL E IDOSOS QUE RESIDEM SOZINHOS EM GRANDES CENTROS URBANOS
}

Yeda Aparecida de Oliveira Duarte'

ORCID: 0000-0003-3933-2179

Luciana Mitsue Sakano Niwa'

ORCID: 0000-0002-9342-7454

Pamella Cristina de Carvalho Lucas' ORCID: 0000-0003-3661-3290

Shirley da Rocha Afonso"

ORCID:0000-003-1824-0451

Daniela Garcia Damaceno III

ORCID: 0000-0001-8656-009X

Suely Itsuko Ciosak'

ORCID:0000-0001-5884-2524

'Universidade de São Paulo. São Paulo, São Paulo, Brasil.

"Centro Paula Souza. São Paulo, São Paulo, Brasil.

"'Universidade do Oeste Paulista. Presidente Prudente, São Paulo, Brasil

Autor Correspondente: Yeda Aparecida de Oliveira Duarte E-mail:yedausp@gmail.com

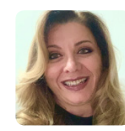

Como citar:

Duarte YAO, Niwa LMS, Lucas PCC, Afonso SR, Damaceno DG, Ciosak SI. Distanciamento social e idosos que residem sozinhos em grandes centros urbanos. In: Santana RF (Org.). Enfermagem gerontologica no cuidado do idoso em tempos da COVID 19. 2.ed.rev. Brasilia, DF: Editora ABEn; 2020.

p 14-19. (Serie Enfermagem e Pandemias, 2). https://doi.org/10.51234/aben.20.e02.c02

\section{INTRODUÇÃO}

Nos últimos meses o mundo tem assistido a evolução de uma pandemia de causa infecciosa denominada Coronavírus Disease 2019 (COVID-19), doença de origem multissistêmica, grave, provocada pelo vírus SARS-CoV-2, de alta transmissibilidade, com maiores taxas de mortalidade entre pessoas idosas, pessoas com doenças crônicas, gestantes e puérperas'.

As pessoas idosas representam cerca de $14,3 \%$ da população brasileira e estão entre os principais grupo de risco. No Brasil, dois grandes fenômenos acompanharam o envelhecimento populacional brasileiro nas últimas décadas: transição demográfica e transição epidemiológica. O primeiro, está diretamente relacionada à diminuição da mortalidade infantil, ao declínio da fertilidade e ao aumento da expectativa de vida e, o segundo, à mudança do perfil de morbimortalidade da população².

Tais mudanças foram acompanhados por novos padrões de comportamento e rearranjos familiares que contribuíram para que parte dos idosos apresentassem comprometimento de sua rede de suporte social, com poucos ou nenhum membro existente ${ }^{3}$. Muitos idosos vivenciam eventos como a morte do cônjuge, levando-os a uma rápida mudança em seu arranjo domiciliar, passando, muitas vezes a residirem sozinhos condição essa que ocorre com $16 \%$ das pessoas idosas residentes no Município de SP ( $\mathrm{n}=290.772$ pessoas) mais predominante entre as mulheres comparativamente aos homens (20,1\% e $10,7 \%$ respectivamente) ${ }^{4}$.

Envelhecer residindo sozinho, também denominados domicílios unipessoais, pode se constituir um uma opção das pessoas idosas pois traz a elas a sensação de liberdade, de controle da própria vida e a expressão máxima de sua autonomia ou, por outro lado, ser o retrato de uma falta de opção, pela inexistência de familiares ou outros membros de uma rede social próxima, o que pode associar-se a diferentes desfechos de saúde, inclusive óbito5-6. 
Dados da Pesquisa Nacional de Saúde (2013) mostraram uma proporção de 15,3\% de idosos morando sozinhos no Brasil, a maioria com comorbidades, dificuldades para a realização de atividades instrumentais de vida diária, piores condições alimentares e maior tempo de comportamento sedentário 7 . Idosos que moram em megalópoles como São Paulo, a sétima maior cidade do mundo, podem tornar-se mais vulneráveis frente a COVID-19.

Segundo a Constituição Brasileira, a Política Nacional do Idoso e o Estatuto do Idoso ${ }^{8}$ é dever da família, da sociedade e do Estado o cuidado das pessoas idosas que assim o necessitarem, mas esse tripé, na prática não é cumprido. Apenas as famílias são responsabilizadas e culpabilizadas em caso de ausência de cuidado. Entre os idosos que residem sozinhos e que não contam com nenhuma rede social de apoio, passa a ser de responsabilidade do Estado e da sociedade civil, as medidas necessárias para garantir seu cuidado em caso de necessidade como, por exemplo, as ações e intervenções nesse momento de pandemia e distanciamento social.

Diante do exposto, é imprescindível refletir e propor ações exequíveis e duradouras para a atenção aos idosos que residem sozinhos em grandes centros urbanos.

\section{OBJETIVO}

Refletir sobre o impacto do distanciamento social imposto pela COVID-19 sobre as condições de vida e saúde das pessoas idosas que residem sozinhas tecendo considerações a respeito das possibilidades e desafios para as políticas públicas vigentes.

\section{MÉTODOS}

Trata-se de um texto teórico-reflexivo sobre o impacto do distanciamento social imposto pela COVID-19 sobre as pessoas idosas que residem sozinhas. As reflexões foram pautadas em publicações internacionais e nacionais relacionados ao tema, dados do Estudo SABE (Saúde, Bem estar e Envelhecimento), estudo longitudinal de múltiplas coortes sobre as condições de vida e saúde das pessoas idosas residentes no Município de SP e vivências das autoras. Os resultados foram categorizados nos seguintes subtemas: Autonomia; Perfil dos idosos que residem sozinhos no Município de SP; Representações sociais do envelhecimento; e Ações solidárias aos idosos em tempos isolamento social pela COVID-19.

\section{RESULTADOS}

\section{AUTONOMIA}

Para um envelhecimento digno e com qualidade é primordial que a pessoa idosa tenha assegurado condições mínimas de existência e possibilidade de participação ativa na sociedade. Nesse sentido, é essencial garantir que a autonomia da pessoa idosa seja respeitada?

Autonomia é expressa pela capacidade de tomar decisões e agir segundo seus próprios valores e crenças, com reconhecimento das possibilidades de atuação própria. Envolve autodeterminação e projeto histórico de individualidade da pessoa. $\mathrm{O}$ avançar da idade não deve ser considerado como um fator incapacitante para que idosos deixem de participar da sociedade ou decidir sobre a condução de própria vida ${ }^{9-10}$.

É frequente se observar, no cuidado em saúde da pessoa idosa, julgamentos equivocados entre a dependência física e o cerceamento da autonomia individual do idoso. Não raro, identifica-se tomadas de decisão que frustram os desejos e o anseios do idoso com justificativas atreladas ao envelhecimento ${ }^{10}$.

Alguns idosos escolhem morar sozinhos porque gostam de manter suas rotinas e por não querer se sentir um peso, optam por não morar com algum outro familiar. Em algumas situações, morar com amigos e parentes pode representar um certo cerceamento da autonomia individual do idoso. 
Morar sozinho, no entanto, não significa dispensar ajuda, quando necessária, para as atividades do dia-a-dia. Pessoas idosas com risco de quedas podem precisar de companhia para caminhar, fazer compras etc. e, precisam compreender a necessidade de aceitação da presença de um acompanhante familiar ou profissional que possa garantir sua segurança. Atualmente, por estarmos vivendo em tempos de desenvolvimento tecnológico, pode-se contar com a ajuda de serviços de telemonitoramento ou teleassistência remotos onde a pessoa idosa ao tocar um determinado dispositivo, aciona uma central e conversa com o atendente que a auxilia ou providencia um serviço de saúde para investigar a demanda e auxiliar a pessoa idosa.

É importante ressaltar que nem todos os idosos optam por morar sozinhos. Há os que moram sozinhos por falta de opção, pois não possuem rede de suporte e não tem a quem pedir ajuda. Nesse contexto, observam-se poucas iniciativas do Estado no sentido de oferecer equipamentos sociais e de saúde, como alternativa para os mesmos. Em outros países existem a possibilidade de escolha por centros-dia, onde a pessoa idosa pode ficar parte ou um período inteiro, fazer sua terapia, receber refeições e aumentar seus vínculos sociais com outros idosos. Podem também, optar por auxílio em casa, onde a comunidade provê as refeições e profissionais/voluntários o auxiliam em sua higienização, limpeza da casa, realização de compras, organização dos medicamentos etc. Podem ainda, optar por morar em repúblicas e instituições de longa permanência para pessoas idosas (ILPIs).

Apesar dos esforços para alavancar as ações políticas voltadas ao idoso, especialmente aquele que vive sozinho, as iniciativas práticas ainda são pequenas, frente a demanda exigida. Se todas as premissas, recomendações e exigências descritas no estatuto do idoso, fossem cumpridas poderíamos ter um cenário diferente para idosos que vivem sozinhos em tempos de pandemia.

Em outras palavras, o direito à autonomia é a capacidade de determinar a forma de viver e conviver na sociedade, percebendo e assumindo as consequências de suas tomadas de decisão. Considerando que, o processo de envelhecimento não é uma condição para a perda da liberdade de escolha da pessoa ${ }^{11}$.

A participação do idoso durante as tomadas de decisões é fundamental para o planejamento do cuidado centrado na pessoa e possibilita o desenvolvimento de uma assistência de saúde ampla e interdisciplinar. Ou seja, o respeito à autonomia do idoso e a garantia da participação na tomada de decisão permite a divulgação das informações coerentes e promove o estabelecimento de opções de tratamentos com pressupostos éticos ${ }^{12}$.

\section{PERFIL DOS IDOSOS QUE RESIDEM SOZINHOS NO MUNICÍPIO DE SÃO PAULO}

Dados da última onda do Estudo SABE - Saúde, Bem estar e Envelhecimento 4 mostraram que, em São Paulo, dos cerca de 300 mil idosos que residem sozinhos nessa cidade, sendo $28,6 \%$ com idade $\geq 80$ anos e $7,8 \% \geq 90$ anos; $63,1 \%$ referem multimorbidades, $47,2 \%$ utilizam polifarmácia, $65,1 \%$ ( $n=189.292$ idosos) estão em processo de fragilização e $26,7 \%$ tem dificuldades no desempenho de atividades básicas de vida diária, necessitando do auxílio de um cuidador, muitas vezes inexistente.

Para vivenciar o distanciamento social imposto pela pandemia, essas pessoas necessitam garantir sua própria subsistência (água, alimentos, higiene pessoal) e o controle de suas doenças crônicas, geralmente feito com o uso de medicamentos. Dessa forma, é necessária uma ajuda externa ou o contato com o meio externo para aquisição de tais itens. Verificou-se que apenas $26,6 \%$ deles ( $n=77.345$ idosos) utilizam computador ou internet e $32,9 \%$ ( $n=95.663$ idosos) não possuem, não utilizam ou tem dificuldade para utilizar telefone (fixo ou celular) o que os coloca fora de contato com o meio externo nas condições de distanciamento social. Soma-se a essas informações que pouco mais de 8,4 mil pessoas idosas referiram não ter a quem pedir ajuda caso necessitassem pois não possuíam uma rede de suporte social que, nesse momento, torna-se um problema a ser resolvido considerando as condições de saúde apresentadas por esse grupo 4 .

Felizmente, vivencia-se que, frente a atual pandemia, a sociedade desenvolveu ações solidárias, de maior ou menor porte, trazendo de certa forma, visibilidade e ajuda a esses idosos solitários, invisíveis e esquecidos. Medidas efetivas que garantam que as necessidades básicas sejam atendidas e medidas de cuidado cheguem até essa população, precisam ser tomadas, para evitar que este grupo, já tão vulnerável e discriminado, passe por essa crise sem ser cuidado. 


\section{AS REPRESENTAÇÕES SOCIAIS DO ENVELHECIMENTO EM TEMPOS DE PANDEMIA DA COVID-19}

Para muitos familiares, sociedade, autoridades sanitárias e políticas, a velhice é representada como um retorno à infância, uma fase de incapacidades. Essa visão relaciona-se, em grande parte às alterações decorrentes do processo de envelhecimento que acarretam limitações funcionais para realizar suas atividades cotidianas. A infantilização da população idosa e o desprezo por suas experiências e histórias pregressas, podem levar os idosos à dependência emocional e, consequentemente, à perda de sua autonomia ${ }^{13}$.

No contexto da pandemia do COVID-19, essa população foi a alvo de destaque, devido às suas vulnerabilidades. Entretanto, verifica-se que a visão estereotipada e estigmatizada divulgada pelas redes sociais, instigou sua ridicularização e o ageísmo. Nesse sentido, embora as ações de proteção à pessoa idosa, assim como as estratégias de distanciamento social e a estratificação de risco sejam positivas para a organização dos serviços, em muitas situações reforçaram a visão preconceituosa acerca dessa parcela populacional com a veiculação de vídeos, fotos e músicas supervalorizando suas características negativas ${ }^{14}$.

Nesse cenário, percebe-se que os conflitos intergeracionais provenientes das medidas de isolamento social impostas a esta população por autoridades e familiares, sem considerar as pluralidades e especificidades do envelhecimento, impactam diretamente em sua saúde física e mental. Assim, além das mudanças na rotina, os efeitos da pandemia se potencializam quando o medo de se sentir sozinho é somado às alterações do processo de envelhecimento ${ }^{14}$.

As notícias sobre a pandemia, o distanciamento/isolamento social, excesso e/ou instabilidade de informações, problemas econômicos e conflitos familiares contribuem com o aumento de crises de pânico, distúrbios do sono e aumento nas taxas de suicídio ${ }^{15-16}$.

As modificações na composição familiar, com a maior participação da mulher no mercado de trabalho, a diminuição das taxas de natalidade e a evidente mudança nos sistemas de valores, contribuíram para as alterações nas relações das famílias e de seus idosos. Dessa forma, o aumento do individualismo, da valorização da vida independente e o enfraquecimento de laços de solidariedade intergeracional, contribui para o aumento de idosos residindo sozinhos ${ }^{17}$.

No contexto da pandemia, o impacto na saúde mental de idosos que residem sozinhos é ainda mais significativo. A falta de convivência com familiares e amigos produzem prejuízos profundos aos idosos, levando-os, muitas vezes, ao desenvolvimento de transtornos depressivos. Por isso, tem sido estimulado o uso de ferramentas tecnológicas, contudo, a população idosa brasileira, majoritariamente, apresenta baixa escolaridade e dificuldade de acesso a essas ferramentas.

Assim, embora se reconheça o potencial de aproximação social proporcionado pela tecnologia, ela possui limitações, sendo necessário o desenvolvimento de estratégias que atendam às necessidades e particularidades dessa população ${ }^{14}$.

Buscar e reinventar formas comunicação como cartas e bilhetes, podem estimular o imaginário dos idosos alfabetizados. Além disso, considerar atividades lúdicas que podem ser desenvolvidas de forma individual e prazerosas com antigos "hobbies" como bordar, crochetar, tricotar, jardinagens e outras artes manuais devem ser estimuladas.

\section{AÇÕES SOLIDÁRIAS AOS IDOSOS EM TEMPOS ISOLAMENTO SOCIAL PELO COVID-19}

A pandemia da COVID-19 tem ampliado o olhar da sociedade para os idosos. É possível identificar, nesse período, experiências exitosas, que empregam esforços a favor da promoção de sua saúde e bem-estar. Inúmeras iniciativas têm ocorrido em todo o país buscando proteger, assistir e acolher os idosos, principalmente os que residem sozinhos. Vizinhos solidários tem se colocado a disposição para as compras, transporte de alimentos e medicamentos. Instituições filantrópicas e religiosas tem se movimentado para prover alimentos preparados, vestimentas, máscaras e álcool em gel para esses idosos. 
Iniciativas de serviços públicos como contato telefônico para monitorar os idosos têm sido, também, realizadas. Entretanto, muitos idosos não tem telefone ou não conseguem manejá-lo adequadamente, sendo necessária a realização de outras abordagens como visitas domiciliárias e ajuda dos vizinhos para garantir o bem-estar do idoso. Apoio social e psicológico realizado por profissionais de saúde, colaboração das universidades, estímulo ao uso de ferramentas tecnológicas, que possibilitam a aproximação social, por meio de mídias sociais, de videoconferência, uso de aplicativos de prestação de serviços, como serviços de orientação médica, monitoramento de atividades entre outros vem sendo empreendidas.

Tais iniciativas são reflexos de uma sociedade que, aparentemente, está se reinventando, se adaptando e se comprometendo, pois, parece compreender a importância em estabelecer relações interpessoais com os idosos, principalmente, entre diferentes gerações. Isto significa uma alteração qualitativa do pensamento colaborativo e compartilhado, observação essa sob a perspectiva do positivismo, que a crise de saúde atual tem demonstrado, na valorização e ressignificação da "cultura do envelhecimento".

\section{CONSIDERAÇÕES FINAIS}

Vivencia-se um momento histórico onde é fundamental minimizar comportamentos individuais e preconceituosos contra os idosos e desenvolver uma consciência social e solidária da sociedade.

Sabe-se, no entanto, que ainda há muito a ser feito para auxiliar os idosos que residem sozinhos e que necessitam de ajuda. Isso vai além desse momento, mas a importância desse, é ter dado visibilidade para essa situação. É fundamental que, a partir de agora, ações estratégicas de transmissão de conhecimentos objetivos, simples e adequados, sejam planejadas para possibilitar a comunicação efetiva com as pessoas idosas além de desenvolver estratégias que possibilitem a inclusão em grupos familiares e sociais diminuindo a solidão e a tristeza, se reinventando a cada dia e buscando motivação para viver.

\section{AGRADECIMENTO}

\section{Ao Departamento Científico de Enfermagem Gerontológica da ABEn Nacional.}

\section{REFERÊNCIAS}

1. Chan JF et al. A familial cluster of pneumonia associated with the 2019 novel coronavirus indicating personto-person transmission: a study of a family cluster. Lancet 2020.

2. Camarano AA. The new demographic paradigm. Ciênc Saúde Colet. 2013;18(12):3446-7.

3. Melo NCV, Teixeira KMD, Barbosa TL, Montoya AJA, Silveira MB. Household arrangements of elderly persons in Brazil: analyses based on the national household survey sample. Rev Bras Geriatr Gerontol. 2016;19(1):139-51.

4. Duarte YAO. SABE Saude, bem-estar e envelhecimento. Estudo longitudinal de múltiplas coortes sobre as condições de vida e saúde dos idosos do município de SãoPaulo- 2015-2019pdf. Escola do Parlamento - Câmara Municipal de São Paulo, 2019.

5. Perseguino MG, Horta ALM, Ribeiro CA. A família frente a realidade do idoso de morar sozinho. Rev Bras Enferm. 2017 ; (2):251-7.

6. $\mathrm{Ng} \mathrm{KH}$. Future of family support: projected living arrangements and income sources of older people in Hong Kong up to 2030. Australas J Ageing. 2016;35(2):113-8.

7. Negrini ELS, Nascimento CF, Silva A, Antunes JLF. Quem são e como vivem os idosos que moram sozinhos no Brasil. Rev. Bras. Geriatr. Gerontol., Rio de Janeiro, 2018; 21(5): 542-550. DOI: https://doi.org/10.1590/1981-22562018021.180101

8. Brasil. Ministério da Saúde. Estatuto do Idoso 3. ed., 2. reimpr. - Brasília : Ministério da Saúde, 2013. 70 p. ISBN 978-85-3341845-5 1.

9. Gama GCN, Mattos MM, Nunes ML. Velhice digna: escolha do tratamento médico. Revista da Faculdade de Direito-RFDUERJ [Internet]. 2016 [citado 2020 Jun 11]; 30:173-195. Disponível em: https://www.e-publicacoes.uerj.br/index.php/ rfduerj/article/view/26903. 
10. Gaspar RB, Silva MM. Zepeda KGM, Silva IR. O enfermeiro na defesa da autonomia do idoso na terminalidade da vida. Rev. Bras. Enferm. [Internet]. 2019 [citado 2020 Jun 11]; 72(6). Disponível em: https://www.scielo.br/pdf/reben/v72n6/pt_00347167-reben-72-06-1639.pdf.

11. Pereira MIT. O direito do idoso à autodeterminação nos cuidados de saúde. Mestrado em Direito. Coimbra: Faculdade de Direito da Universidade de Coimbra; 2018 [citado 2020 Jun 11]. 143 p. Disponível em: https://estudogeral.sib.uc.pt/ bitstream/10316/85820/1/Disserta\%c3\%a7\%c3\%a3o\%20final\%20pdf.pdf.

12. Paranhos DGAM, Albuquerque A. A autonomia do paciente idoso no contexto dos cuidados em saúde e seu aspecto relacional. R. Dir. sanit. [Internt]. 2018 [citado 2020 Jun 12] 19(1): 32-49. Disponível em: https://www.revistas.usp.br/rdisan/ article/view/148123/141735

13. Santos RAAS, Corrêa RGCF, Rolim ILTP, Coutinho NPS. Atenção no cuidado ao idoso: infantilização e desrespeito à autonomia na assistência de enfermagem. Revista Pesquisa em Saúde. 2016 sep/ dec; [cited 2020 jun 13]; 17(3):179-83. Available from: http://www.periodicoseletronicos.ufma.br/index.php/revistahuufma/article/view/6793/4335

14. Hammerschmidt KS de A, Santana RF. Saúde do idoso em tempos de pandemia Covid-19. Cogitare enferm. [Internet]. 2020 [cited 2020 jun 13]; 25. Disponível em: http://dx.doi.org/10.5380/ce.v25i0.72849.

15. Roy D, Tripathy S, Kar SK, Sharma N, Verma SK, Kaushal V. Study of knowledge, attitude, anxiety \& perceived mental healthcare need in Indian population during COVID-19 pandemic. Asian Journal of Psychiatry, 2020. 51. 102083. Disponível em: https://doi.org/10.1016/j.ajp.2020.102083

16. Vahia IV, Blazer DG, Smith GS, Karp JF, Steffens DC, Forester BP, Tampi R, Agronin M, Jeste DV, Reynolds III CF. COVID-19, Mental Health and Aging: A Need for New Knowledge to Bridge Science and Service. Am J Geriatr Psychiatry (article in press) 2020. Disponível em: DOI: https://doi.org/10.1016/j.jagp.2020.03.007

17. Carrara BS, Espírito Santo PMF. Velhice institucionalizada em tempos pós-modernos: a identidade em universo paralelo? REv.enferm.UFPE online;10 (5):1672-1684 